\title{
Investigation of a Digital Hydraulic Actuation System on an Excavator Arm
}

\author{
Alessandro Dell'Amico, Marcus Carlsson, Erik Norlin, and Magnus Sethson \\ Division of Fluid and Mechatronic Systems, Department of Management and Engineering, Linköping University, Linköping, \\ Sweden \\ E-mail: alessandro.dellamico@liu.se, magnus.sethson@liu.se
}

\begin{abstract}
Digital hydraulics is an ongoing trend that offers many interesting advantages and possibilities. Digital refers to that the system output is discrete, e.g. using an on/off valve with only discrete values or a finite amount of flow steps available. The advantages mentioned when compared to analogue systems are better performance, robust and fault tolerant, and amplitude independent bandwidth. On the other side noise and pressure pulsations must be handled, the physical size can be a problem, and the system requires complicated control. When considering control of linear motion, there are mainly two branches, controlling the flow with several parallel connected on/off valves, which generates discrete output flow values, or switching valves, which in theory can generate any mean output flow. The latter only requires one valve for each flow path but the demand for fast valves is very high, while the former requires many valves but avoids high frequent switching. With the introduction of a multi-chamber cylinder, secondary control is now also possible for linear motion. This paper is a first step in the investigation of the system applied to an excavator arm. The cylinder has four chambers, each with different area. Three pressure lines are used and a valve-pack of 27 on/off valves. The valve-pack connects the three pressure lines with each chamber generating 81 available force steps. The scope has been to start out with relative simple control of the velocity of the cylinder. To handle unnecessary switching of valves, different penalty strategies were tested. The results are promising where relatively smooth control could be achieved at the same time challenges with the system were identified. Next step is to investigate the force transients due to different capacitance in all four chambers as well as mode control for better accuracy. Energy potential compared to original system remains to investigate as well.
\end{abstract}

Keywords: Digital hydraulics, secondary control, excavator, on/off-valves

\section{Introduction}

Digital hydraulics is a promising technology for flow control. It has gained extensive research efforts over the latest years. Digital refers to the output of the system being discrete. For valve flow control there are two main branches, one is the use of several parallel configured on/off valves replacing each and one of the control edges in an conventional proportional valve. The other strategy makes use of a modulating technology. Both technologies are based on on/off valves but the latter only requires one valve for each control edge, [1]. However, there is a demand for very fast and durable valves. These are challenges that need to be handled as well as parasitic losses due to large capacitances of the hydraulic system and pressure peaks. The former technique avoids fast continuous switching of valves but the physical size of the unit becomes large due to the many valves involved, [2]. In this case each Digital Flow Control Unit (DFCU) generates a discrete flow output. The benefits are a robust and possible fault tolerant solution, amplitude independent opening, and flexibility. The flexibility of the system allows for different control modes and when compared to traditional load sensing system, high energy savings are reported, [3]. However, the solution should also be compared to other solutions with distributed valves, like in [4]. The main challenges with parallel configured valves are; system size and cost, complex control strategies and noise and pressure pulsations. The pressure pulsations are due to the uncertainty in valve timings and is dependent on the coding of the DFCU [5]. The performance of the system is dependent on the characteristics of the on/off valves, among others. In many cases commercial valves are not sufficient and research has been focused on development of high performance valves, [6]. The need for fast valves and advanced controllers is shown in [7] and [8], where a digital hydraulic system is compared to a servo valve system for different applications.

With the development of a multi-chamber cylinder, [9], secondary control is now possible for linear actuator control. Secondary control has previously been restricted to displace- 
ment machines only. The technique has a high potential of being a very energy efficient system [10], since no valve throttling takes place. The system uses a constant pressure supply line and if an accumulator is connected to the system, energy can be recovered during retardation of the load. Several secondary control units can be added to the same supply thereby simplifying the piping. A secondary controlled swing drive of an excavator was investigated in [11] and showed upon a possible $60 \%$ energy consumption reduction. The challenge with secondary control is however the need for advanced controllers, [12]. For linear motion a multi-chamber cylinder is used together with constant pressure supply lines. A valvepack with on/off valves combines the supply lines with the different chambers. In this way the cylinder delivers discrete force steps. In [13] a four chamber cylinder is investigated with two pressure lines for position control. That system has 16 possible force levels to control the movement of a load. A PI-controller is used together with a switch strategy that minimizes unnecessary switching. The results showed a good controllability of high inertia loads and a reduction of $60 \%$ in energy consumption compared to a traditional LS system for the same application.

The scope of this paper is to investigate a four chamber cylinder with three pressure lines, generating 81 force levels, applied to an excavator arm. This is a first attempt in trying to understand the system characteristics of digital hydraulics, studying the advantages and the challenges. At first only the boom of the arm is installed and a simple control structure for velocity control is tested.

\section{System setup}

A test system has been formed to provide a realistic environment for laboratory tests. In the beginning, only the inner arm is installed. It is going to be extended with the complete excavator arm. With two arms later installed, the system can also be tested for over-centre loads as well as energy recovery from two actuators.

An overview of the system is seen in fig. 11 The main components of the system is the excavator arm, the hydraulic supply system with its three pressure lines, the 27 -valves block, and the multi-chamber cylinder. The cylinder is controlling the motion of the the boom of the excavator arm. The supply system is at this point rather simple with focus only on functionality. Three pressures are used, 200, 110, and 20 bars. The highest pressure is set by a pressure relief valve (Bosch Rexroth DBDS10K1X/315) and the pump running at constant speed. The medium and low pressure are in turn set by two pressure reducing valves (Bosch Rexroth DR20G54X/200YM and DR20G5-4X/100YM). Two relief valves are also installed for security at medium and low pressure line (Bosch Rexroth DBDS10G1X/200 and DBDS10G1X/100). The actual pressures in the three lines are of course affected by the characteristics of the relief and reducing valves. Three accumulators of 4 litres each are installed at the three pressure lines. They are not sufficiently large for energy recovery operation but will smoothen out the supply pressures.

The valve-pack consists of 27 on/off valves with $50 \mathrm{~ms}$ rated

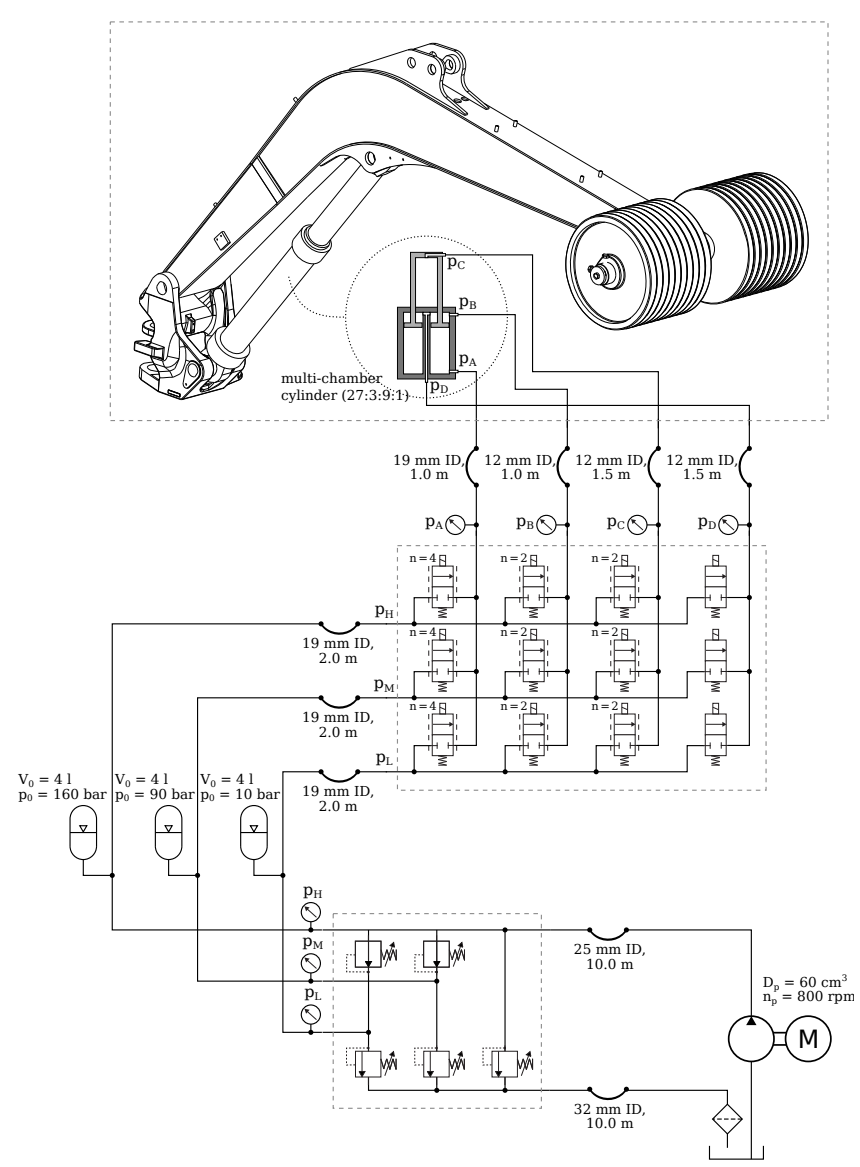

Figure 1: System overview.

response time. The valve-pack connects the pressure lines to each chamber of the cylinder. The connection between each pressure line and each chamber is done by several parallel configured valves. The largest chamber has four valves in parallel for each pressure line and smallest has only one valve for each pressure line. The other two chambers have two valve each for each pressure line. To use several parallel connected valves for the chambers is beneficial in several ways. The flow demand for a given velocity of the piston is different for each chamber. To decrease the pressure drop a larger effective area is achieved by several valves. Another advantage is the possibility to control the pressure build up in a more effective way. The pressure build up is dependent on the flow in or out of the chamber and the capacitance of the chamber. A more robust solution is also achieved with several valves. If one valve breaks the arm can still be operated, at least for the three larger cylinder areas.

The cylinder consists of four chambers with different areas with relative relation 27:9:3:1. Two chamber extends the cylinder and two chambers retracts it. By combining the three pressures in the four chambers of the cylinder $3^{4}=81$ force steps can be applied by the cylinder. An overview of the cylinder is seen in fig. 2 Figure 3 shows the available force distribution. The maximum available force is $129.9 \mathrm{kN}$ and the maximum retraction force is $2.1 \mathrm{kN}$. Since only one arm is in place at the moment an axle is installed at the tip of the boom. 
In this way the boom can be loaded with up to $400 \mathrm{~kg}$.

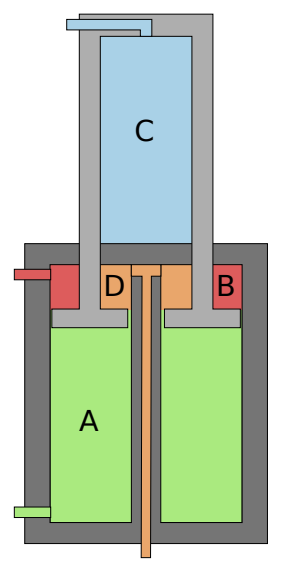

Figure 2: An overview of the multi-chamber cylinder.

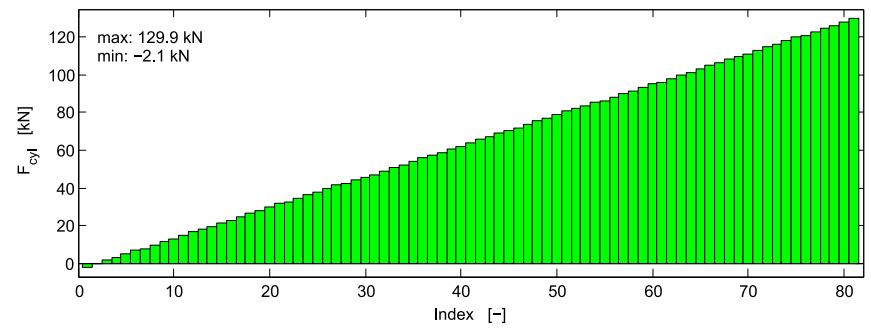

Figure 3: Available force spectrum for $H P=200$ bar, $M P=$ 110 bar, and $L P=20$ bar.

The valve-pack is controlled by an prototype Exertus HCMseries unit which communicates with the industrial computer MPL PIP8 via a CAN bus. The controller software is developed in Matlab/Simulink and downloaded to the industrial PC through xPC Target. The valve control unit also provides analogue input channels for sensor data. Sensors used are pressure sensors for the three pressure lines and each chamber of the cylinder. In this way the pressure drop over the valve-pack can be measured. The position of the piston is measured with a potentiometer multi-turn wire sensor. The derivative of the position signal is filtered with a second order low pass filter to get the velocity of the piston.

Future work of the test rig involves the installation of the outer arm as well. This will further extend the possibilities of the elaborative studies of the system characteristics. The multichamber cylinder for the outer arm will use the same three pressure lines as the installed boom cylinder. The amount of hoses is reduced compared to a traditional system. Also, energy can be recovered from both actuators. The future test rig is shown i fig. 4 .

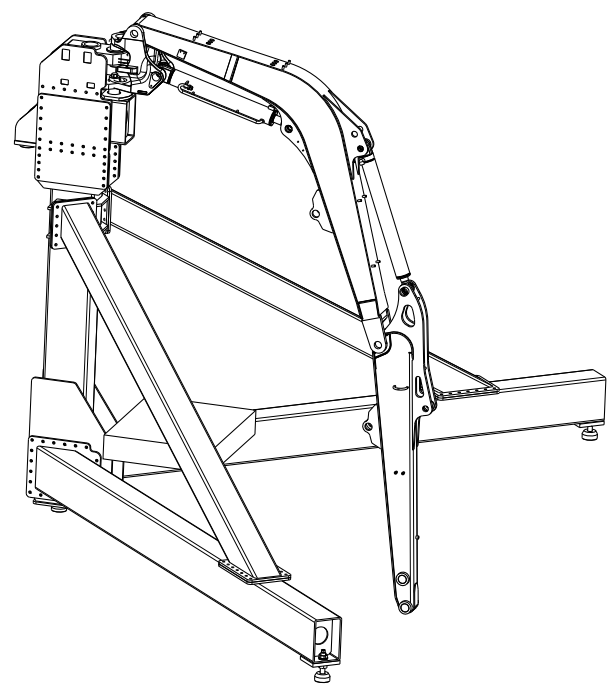

Figure 4: Future test rig of the crane.

\section{Control structure}

A traditional hydraulic system gives the operator a way to control the velocity of the piston. The system studied is secondary controlled and is therefore force controlled. Some kind of external controller is therefore required in order to control the velocity. In order to investigate the system and its behaviour a simple control approach has first been tested. The actual force applied by the cylinder is calculated as:

$$
F=p_{A} A_{A}-p_{B} A_{B}+p_{C} A_{C}-p_{D} A_{D}
$$

\subsection{Control Strategy A}

The controller is a PI-controller that compares a reference velocity of the piston with the actual velocity. The output from the controller is the required force to follow the reference. Since the system is digital and therefore discrete, only a finite amount of force steps are available and the closest available force compared to the reference force needs to be chosen. This is done by comparing the reference force with each available force and the combination that gives the smallest error is chosen according to 2

$$
u=\min \left(\left|F_{r e f}-\hat{F}\left(u_{i}\right)\right|\right) \quad i=1: 81
$$

The actual available force steps depends on the pressure in the three supply lines. As they will fluctuate during movement of the crane, the supply pressures are measured and the force distribution updated each time step. The control structure is seen in fig. 5. The measured supply pressures are quantized to avoid influences from measurement noise. 


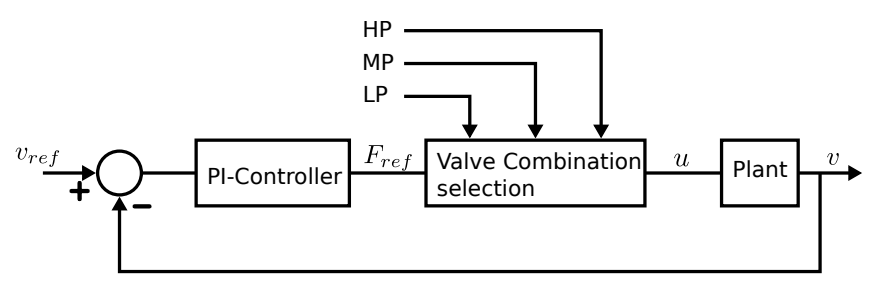

Figure 5: Controller structure.

Due to the discrete nature of the system, unnecessary switching between different force states might occur. To avoid this a penalty for switching can be introduced. The controller is only allowed to change force state if changing state has big enough effect. The penalty is added to every force level except the previous one. This is according to [13]. The selection of combination can now be updated as follows.

$$
\begin{gathered}
u=\min \left(\left|F_{r e f}-\hat{F}\left(u_{i}\right)\right|+P j\right) \quad i=1: 81 \\
j= \begin{cases}1 & \text { if } u_{i} \neq u_{\text {previous }} \\
0 & \text { if } u_{i}=u_{\text {previous }}\end{cases}
\end{gathered}
$$

where $P$ is the penalty and is a tuning variable together with the PI-controller gains. $u$ and $u_{\text {old }}$ are the new and old control combination respectively.

\subsection{Control Strategy B}

The other strategy also tested uses a score-based system to avoid frequent switching of high amplitude pressure changes. High amplitude changes will have biggest effect on the smoothness, especially if it occur on all chambers at the same time. A high amplitude change is rated high in score and a low amplitude change rated low. For each change in force step a total score can be calculated as the sum of the scores from all chambers. If the total score is high a penalty is added to the previous force step in order avoid changing back to it. Again, this mimics eq. 3 .

\section{Experimental setup}

Several tests are conducted in order to evaluate the system's ability to be controlled by a velocity reference. For all tests the input signal is a pulse train with an amplitude of $3 \mathrm{~cm} / \mathrm{s}$. The tests are carried out in both directions of the cylinder. Before changing direction the arm is set to stand still for 2 seconds. The input reference signal is somewhat smoothen by limiting the maximum allowed acceleration and jerk. For each control structure two load cases are tested, $200 \mathrm{~kg}$ and $400 \mathrm{~kg}$. All controller parameters are tuned experimentally and a penalty of $2000 \mathrm{~N}$ is used.

\section{Results}

Figure 6 and fig. 9 show the results of the test with $200 \mathrm{~kg}$ and $400 \mathrm{~kg}$ respectively. For the $200 \mathrm{~kg}$ weight only the B and D chambers switch pressure. They are small which results in a quiet smooth movement. For the heavier weight all chambers changes pressure. This occurs frequently during acceleration.
Since also the larger chambers changes pressure the movement is less smooth. Figure 7 shows the result of the test with $200 \mathrm{~kg}$ and penalty function A. Frequent switching is no longer taking place but accuracy has become worse which affected the smoothness. Figure 10 shows the same run with $400 \mathrm{~kg}$. Here it is even more obvious that the frequent switching no longer takes place. The movement is smoother due to the larger inertia but since no functionality is implemented to handle zero velocity input, the resolution is not high enough to find force equilibrium for those cases. Figure 8 shows the test with strategy B. No difficult pressure changes occur and the result is similar as in the case with no penalty function implemented. The effect of strategy B is seen in fig. 11 . where frequent switching of all chambers at the same time is avoided.
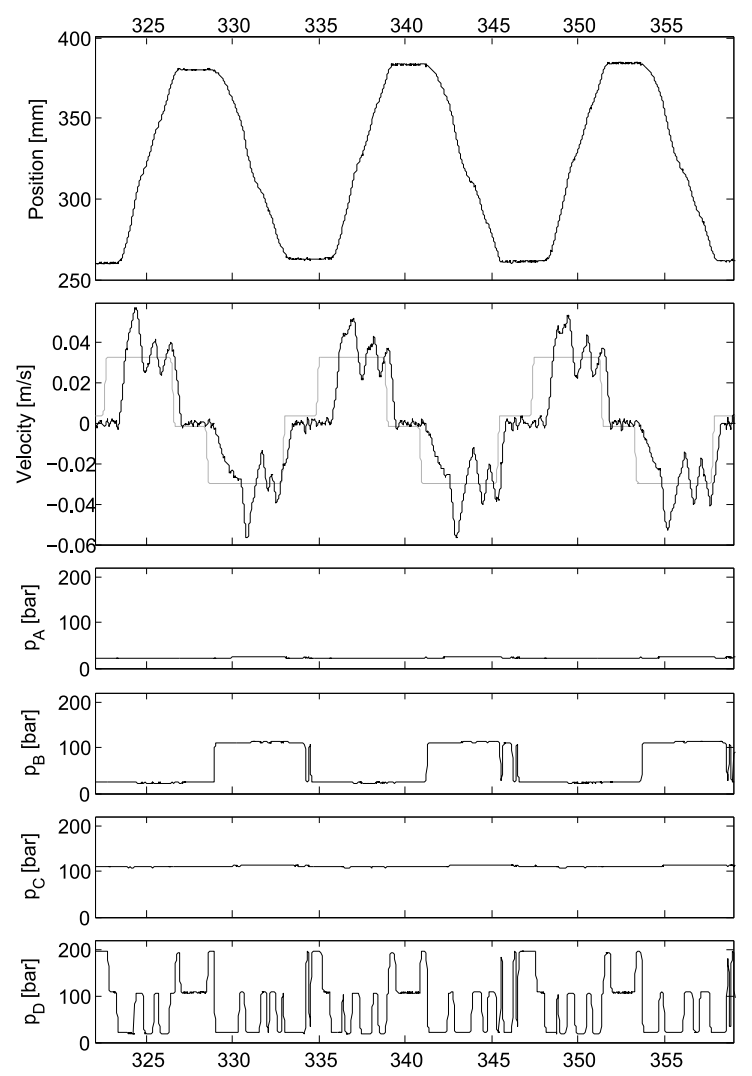

Figure 6: $200 \mathrm{~kg}$ with no penalty. 

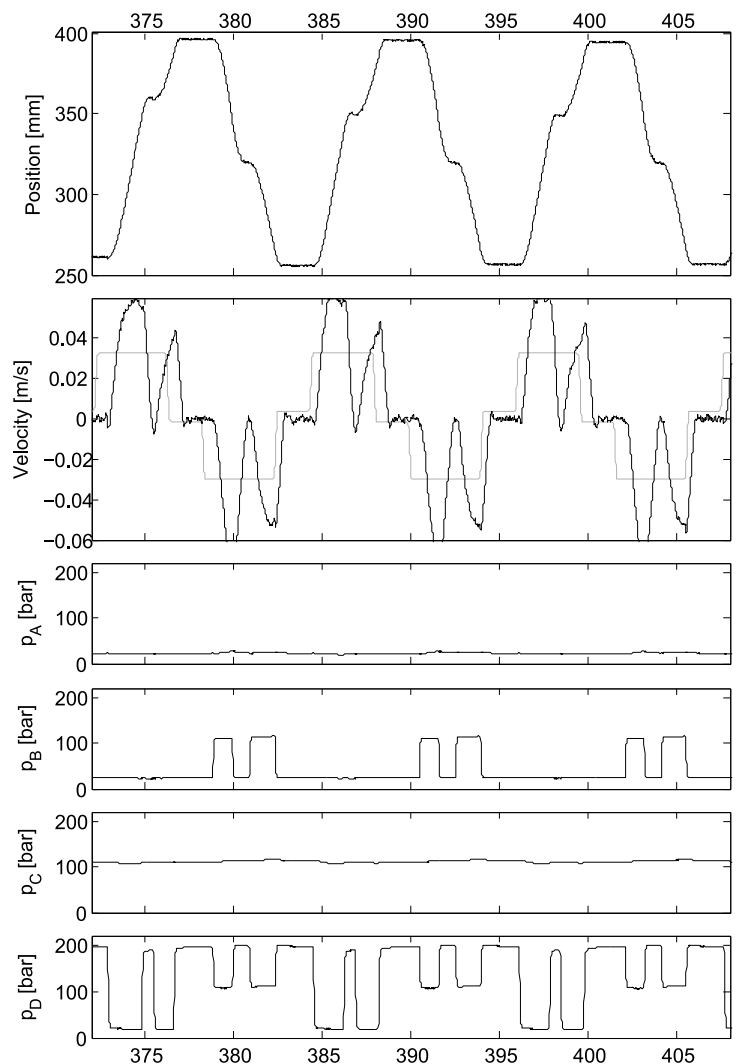

Figure 7: $200 \mathrm{~kg}$ with penalty strategy A.
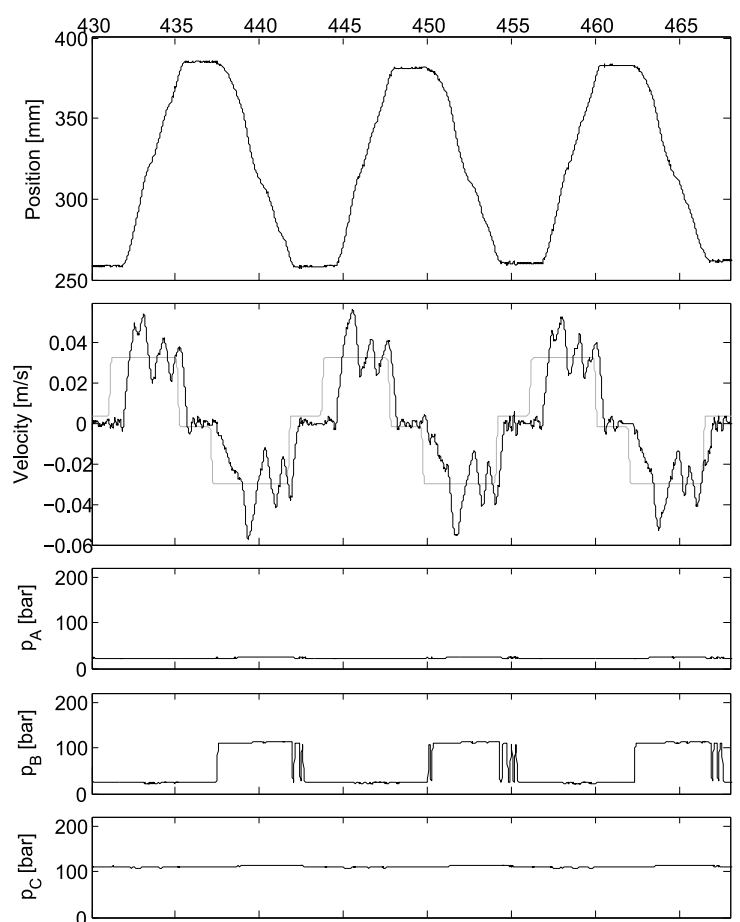

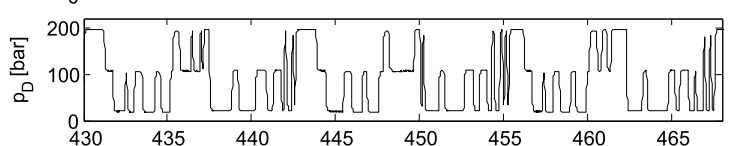

Figure 8: $200 \mathrm{~kg}$ with penalty stategy $B$.
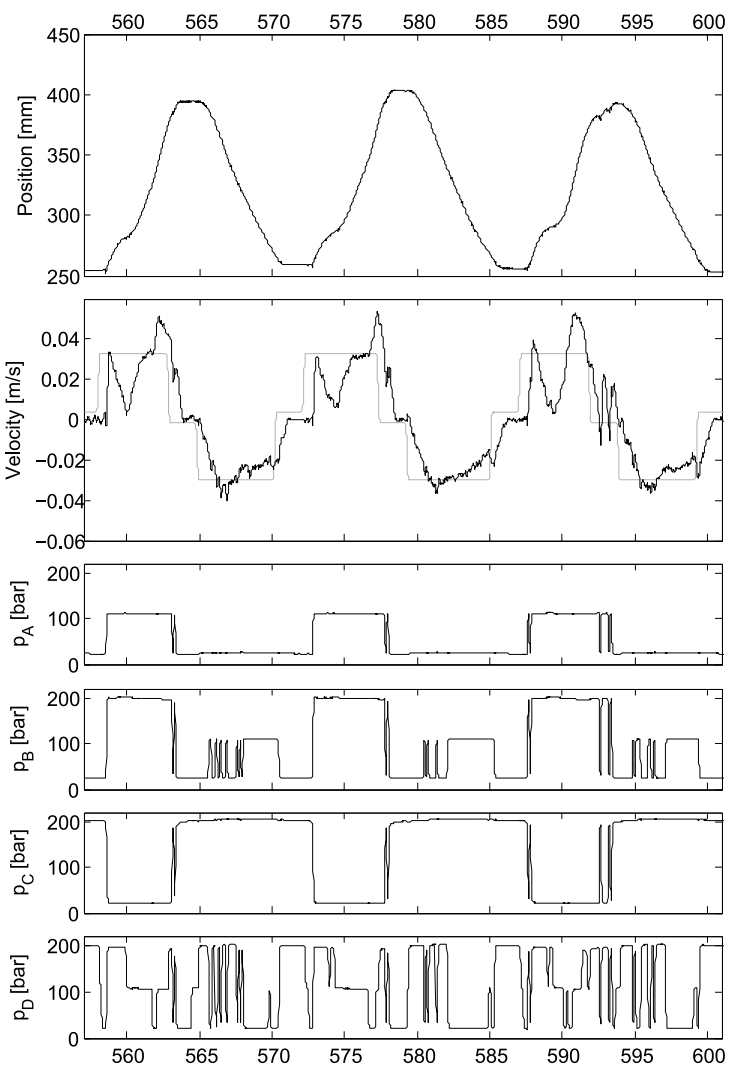

Figure 9: $400 \mathrm{~kg}$ with no penalty.
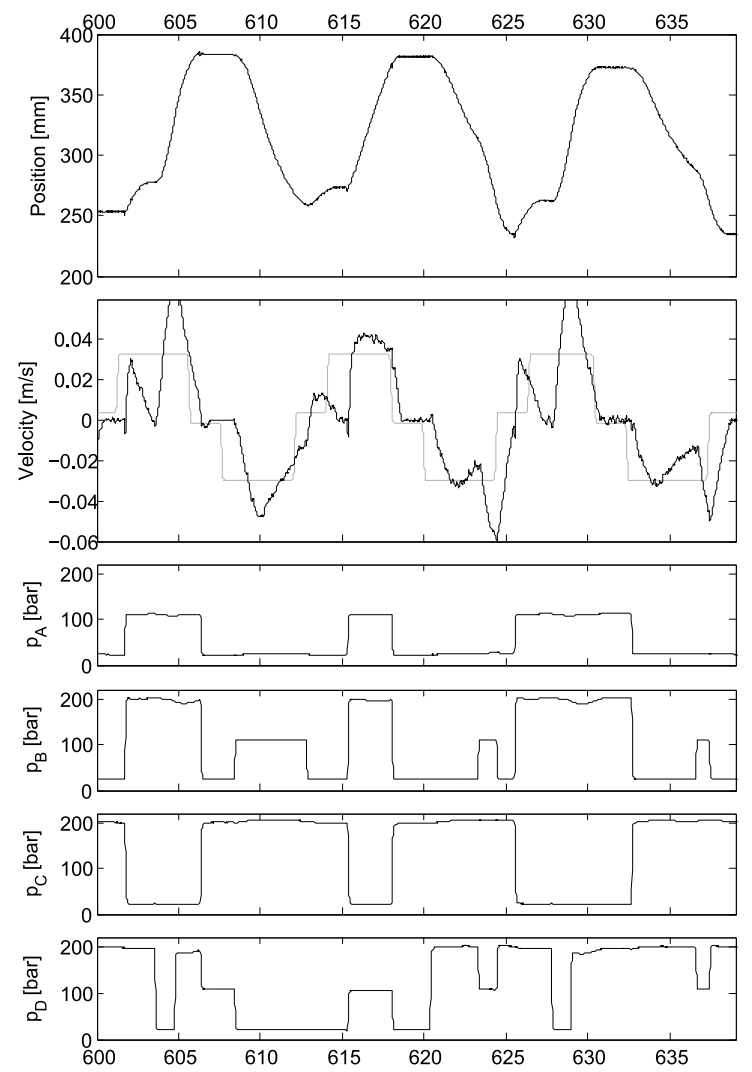

Figure 10: $400 \mathrm{~kg}$ with penalty strategy A. 

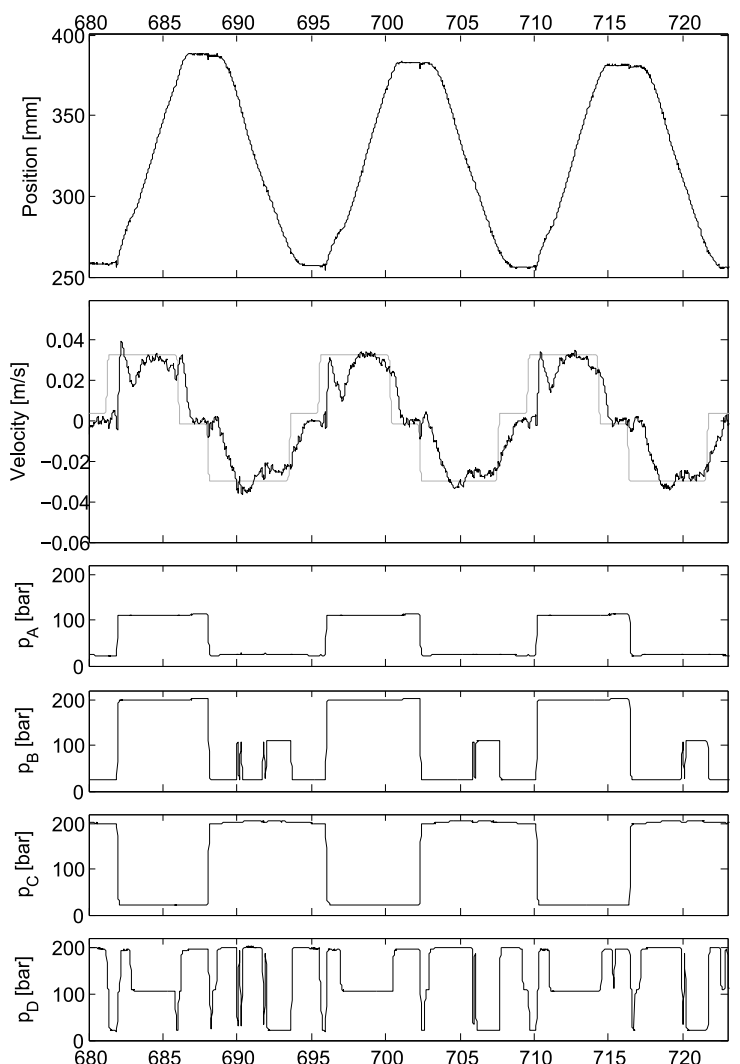

Figure 11: $400 \mathrm{~kg}$ with penalty strategy B.

\section{Discussion and future work}

The results from the tests are promising. They show that with simple control a relatively smooth operation of the arm is possible. Due to the nature of the system frequent switching between force steps can occur and must be handled in some way. The different strategies tested showed that there is a compromise between accuracy, smoothness of the arm and frequent switching. Another factor is the energy efficiency. This has not been studied in this work but it was shown in [13] that the biggest losses are the compressibility losses and this should be considered as well when selecting switching strategy. Next step is to add the outer part of the excavator arm and study the energy consumption compared to the original system to get an idea of the energy saving potential. Another thing to be studied is the effect of different pressure rates in the chambers due to different capacitances. This could lead to force transients that counteracts the movement of the arm. Noise is another concern that should be studied and is related to the pressure build up in the chambers and frequent switching.

\section{Conclusions}

A test rig of an excavator arm actuated by a secondary controlled multi-chamber cylinder has been built and tested. At this first stage only the boom is installed and simple control strategy is implemented and evaluated. For a lighter load the big chamber do not switch anything and the effect of switching in the smaller chambers is negligible on the smoothness. For the heavier weight all chambers switches pressure more frequent which affected the smoothness of the arm. Two different strategies were tested to avoid this behaviour, both affecting the switching of the valves. By adjusting the control and switching strategies it is potentially possible to adjust operation conditions, controller trajectory performance and noise generation.

\section{References}

[1] Rudolf Scheidl and Bernhard Manhartsgruber. State of the art in hydraulic switching control - components, systems, applications. In The Ninth Scandinavian International Conference on Fluid Power, Linköping, Sweden, June 1-3 2005.

[2] Matti Linjama. Digital fluid power - state of the art. In The Twelfth Scandinavian International Conference on Fluid Power, Tampere, Finland, May 18-20 2011.

[3] Matti Linjama, Mikko Houva, Pontus Boström, Arto Laamanen, Lauri Siivonen, Lionel Morel, Marina Waldèn, and Matti Vilenius. Design and implementation of energy saving digital hydraulic control system. In The Tenth Scandinavian International Conference on Fluid Power, SICFP'07, Tampere, Finland, May 21-23 2007.

[4] Björn Eriksson. Mobile Fluid Power Systems Design. $\mathrm{PhD}$ thesis, Linköping University, 2010.

[5] Arto Laamanen, Matti Linjama, and Matti Vilenius. On the pressure peak minimization in digital hydraulics. In The Tenth Scandinavian International Conference on Fluid Power, SICFP'07, Tampere, Finland, May 21-23 2007.

[6] Matti Linjama and Matti Vilenius. Digital hydraulics - towards perfect valve technology. In The Tenth Scandinavian International Conference on Fluid Power, SICFP'07, Tampere, Finland, May 21-23 2007.

[7] Matti Linjama, Juho Seppälä, Jouni Mattila, and Matti Vilenius. Comparison of digital hydraulic and traditional servo system in demanding water hydraulic tracking control. In Fluid Power and Motion Control, 2008.

[8] Ville Ahola, Matti Linjama, and Matti Vilenius. High performance digital hydraulic servo system for linear cyclic motion. In The Tenth Scandinavian International Conference on Fluid Power, SICFP'07, Tampere, Finland, May 21-23 2007.

[9] Ari Sipola, Hannu-Pekka Vihtanen, Matti Linjama, and Arto Laamanen. Digital hydraulic system, 2010.

[10] Göran Palmgren and Karl-Erik Rydberg. Secondary controlled hydraulic systems - energy aspects and control strategies. In International Conference on Fluid Power, Tampere University of Technology, Finland, March 24-26 1987. 
[11] Karl Pettersson and Seppo Tikkanen. Secondary control in construction machinery - design and evaluation of an excavator swing drive. In The 11th Scandinavian International Conference on Fluid Power, SICFP'09, Linköping, Sweden, June 2-4 2009.

[12] H. Berg and M. Ivantysynova. Design and testing of a robust controlller for secondary controllled hydraulic drive. Proceedings of the Institution of Mechanical Engineers, 213:375-386, 1999.

[13] M. Linjama, H-P. Vihtanen, A. Sipola, and M.Vilenius. Secondary controlled multi-chamber hydraulic cylinder. In The 11th Scandinavian International Conference on Fluid Power, SICFP'09, Linköping, Sweden, 2009. 